\title{
Del distrito industrial al distrito rural: implicaciones teóricas para el desarrollo territorial
}

\author{
Juan Sebastián Castillo ${ }^{1}$ y María del Carmen García ${ }^{2}$
}

RESUMEN: Derivando del tradicional Distrito Industrial Marshalliano, el Distrito Rural se introduce como nuevo concepto para el desarrollo territorial debido al cambio cualitativo poblacional que desde 1970 se produce en los países desarrollados y desde los años ochenta en España y Castilla-La Mancha. Las zonas urbanas perdieron peso y la jerarquía urbana y las relaciones centro-periferia cambiaron hacia otras menos escalonadas y multipolares. En el medio no-urbano surge el movimiento rural-rural, de los núcleos de menor dimensión hacia las cabeceras comarcales y/o núcleos de mayor población más próximos, en una reproducción de un modelo centro-periferia en el nivel territorial rural.

PALABRAS CLAVES: Distrito rural, economías de escala sociales, metodología ISTAT, modelos hedónicos poblacionales.

JEL classification: C01, J11, R00.

From industrial district to rural district: Theoretic implications for territorial development

\begin{abstract}
As opposed to the traditional Marshallian Industrial District, the Rural District becomes a new concept for territorial development thanks to the qualitative changes of population occurring since 1970 in developed countries and since 1980 in Spain and Castilla-La Mancha. The urban areas are decreasing in importance and the urban hierarchy and center-periphery relations evolved into less hierarchic and multipolar relations. Rural-rural movements occurred in rural environments, from minor cores to region cores and/or higher populated cores as a reproduction of a center-periphery model at rural territorial level.
\end{abstract}

KEYWORDS: Rural district, social-scale economy, ISTAT methodology, population hedonic models.

Clasificación JEL: C01, J11, R00.

\footnotetext{
Instituto de Desarrollo Regional y ETSIA. Universidad de Castilla-La Mancha. Campus universitario s/n, 02071 Albacete.

2 Instituto de Desarrollo Regional. Universidad de Castilla-La Mancha. Campus universitario s/n, 02071 Albacete.

Dirigir correspondencia a: María del Carmen García. E-mail:mariacarmen.gcortijo@uclm.es.
}

Recibido en mayo de 2011. Aceptado en octubre de 2011. 


\section{Un nuevo concepto de aproximación al desarrollo rural: El Distrito Rural}

En los países desarrollados, desde 1970, y en España, ya avanzada la década de los ochenta, se empezó a experimentar un nuevo proceso poblacional y demográfico: las zonas urbanas perdieron peso relativo y jerarquía y las relaciones centro-periferia cambiaron hacia otras menos escalonadas y multipolares. Castilla-La Mancha no fue indiferente a este acontecer donde perdía fuerza la visión de una sociedad rural que se despoblaba de forma generalizada. Actualmente se encuentra en una fase de recuperación poblacional; la salida rural global disminuye su intensidad y se pone de manifiesto que el tradicional proceso de pérdida rural, en los núcleos más pequeños, está siendo compensado con otro proceso inverso de emigración urbana hacia otro ámbito (corredores) y, sobre todo, por el predominio y consolidación de movimientos rural-rural en una dinámica centro-periferia, donde el centro es un municipio mayor de la misma comarca o muy próximo geográficamente y la periferia está constituida por los municipios más pequeños que pivotan alrededor.

La nueva dinámica se confirma, cuantitativamente, con la técnica del ajuste analítico. Esta consiste en determinar la tendencia de una variable ajustándola a una función matemática. Analíticamente, su expresión es:

$$
Y_{i}=f(t)+u_{i}
$$

donde $Y$ es la serie a estudiar, $f$ representa la forma funcional en que se relacionan la serie $Y$ y la serie de tiempo $t$, u es la perturbación aleatoria.

En esta situación la variable Y representa el peso de la población de los municipios periféricos, de los municipios centro y de las capitales de provincia en el total de Castilla-La Mancha. Atendiendo al número de habitantes se consideran municipios periferia aquellos con una población inferior a los 4.000 habitantes y municipios centro los que igualan o superan esa cifra, excluyendo las cinco capitales de provincia. Tras representar las tres series (Gráfico 1), se observa un punto de inflexión en 1980: la población de los municipios centro supera la de los municipios periferia y la población de las capitales de provincia se empieza a estancar, produciéndose incluso un descenso a partir del 2000. Así analizamos la tendencia de dos periodos: 1950-1970 y 1980-2010, ajustando las series con una regresión de tendencia lineal, $Y_{i}=a+b t+u_{i}$ resultando, el valor estimado de crecimiento de la población $(\hat{b})$. En la primera etapa las capitales de provincia fueron las únicas en aumentar su población a un ritmo del $2,45 \%$ mientras que el resto de los municipios perdían población. Es a partir de la década de los 80 cuando los centros también crecen $(2,3 \%)$ y por encima de las capitales, que sólo lo hacen un 0,4\%. Finalmente se observa que en el periodo 2000-2010 sólo los municipios centro reciben población (6\%). 


\section{GRÁFICO 1}

\section{Evolución peso de la población: Municipios periferia, centro y capitales}

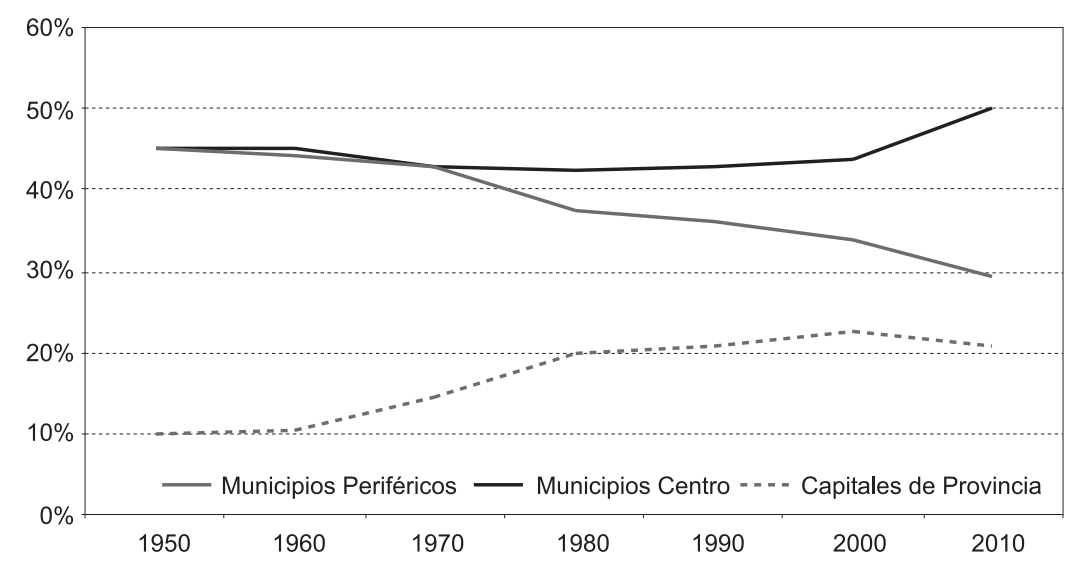

Fuente: Elaboración propia con datos INE.

\section{CUADRO 1}

Tendencia de la población de: Municipios periferia, centro y capitales

\begin{tabular}{lcccccc}
\hline \hline & \multicolumn{2}{c}{ Municipios Periféricos $(<\mathbf{4 0 0 0} \mathbf{h})}$. & Municipios Centro $(\mathbf{4 0 0 0} \mathbf{h})$. & \multicolumn{2}{c}{ Capitales de Provincia } \\
\hline $1950-1970$ & $-0,011$ & $(0,97)$ & $-0,013$ & $(0,74)$ & 0,0245 & $(0,86)$ \\
\hline $1980-2010$ & $-0,0268$ & $(0,94)$ & 0,0229 & $(0,73)$ & 0,0039 & $(0,22)$ \\
\hline $2000-2010$ & $-0,0442$ & $(1)$ & 0,0622 & $(1)$ & $-0,0179$ & $(1)$ \\
\hline \hline
\end{tabular}

Notas: Entre paréntesis aparece el coeficiente de determinación de las ecuaciones estimadas.

Fuente: Elaboración propia con datos INE.

Estos primeros resultados corroboran lo afirmado. En nuestra opinión, las razones y fuerzas motrices de este cambio cualitativo, están vinculadas a la aparición y reproducción de mecanismos explícitos dentro de la "Nueva Geografía Económica" de Krugman (1991, 1992), pero sobre bases sociales en lugar de económicas, lo que le confiere un verdadero rasgo característico y novedoso y, sobre todo, conceptualmente, una dimensión teórica atractiva: la conformación de los Distritos Rurales. Entendidos como una evolución especifica del concepto de distrito industrial de origen marshalliano, con una base social, en el caso del Distrito Rural, en las economías de escala centrípetas en lugar de la base productiva presente en los distritos industriales.

Los Distritos Rurales se definirían como aglomeraciones rurales de población identificadas en entidades socioterritoriales caracterizadas por la presencia activa de población y de actividad económica en una zona rural de dimensión media, con un sistema de valores común que se difunden a todo el distrito a través de lo consuetu- 
dinario y el entramado institucional, bien derivado de la comarcalización, o bien de territorios vehiculados en torno a los Grupos de Acción Local ${ }^{1}$. Este nuevo término constituye una sedimentación de un proceso de reorganización demográfica y cambio sectorial y de la configuración productiva. También se involucra a un conjunto de políticas, líneas de acción, medios instrumentales y proyectos, que buscan actuar sobre la organización espacial para diseñar, en el largo plazo, una estructura del territorio que integre de manera armoniosa y gradual los componentes de población, recursos naturales e infraestructura dentro del contexto del desarrollo humano sostenible y el equilibrio entre los procesos de desarrollo endógeno y exógeno.

Ahora bien, en este sentido es necesario circunscribir una base geográfica y socioeconómica que recoja las características de un Distrito Rural. Como señalan Boix y Galletto (2005), los límites administrativos regionales son demasiado grandes para albergar un concepto como el del Distrito Industrial. Además el Distrito, normalmente, se extiende entre varios municipios, por lo que su utilización también resulta inapropiada. El problema se resuelve considerando una división territorial inferior a la región, superior a la municipal e integrada por municipios con características geográficas similares y semejanzas en sus modos de vida: a priori parecen apropiados los Grupos de Acción Local (GAL). Adicionalmente, como señala Bielza de Ory (2003), los GAL responden a un principio de cohesión de sus poblaciones, son de carácter funcional y sistémico, se definen por su función, por su organización, por la existencia de algo que ordena las partes en el todo; configurando un sistema de relaciones con mayor cohesión en su interior que las existentes con las poblaciones del exterior. Son delimitaciones territoriales vinculadas a la política de desarrollo rural de la UE, acotaciones no definidas por criterios administrativos, sino de carácter natural y cultural y que concentran población y trabajadores en los municipios que actúan de cabecera (centro); como se demuestra, seguidamente, con un análisis estadístico poblacional y con la metodología del ISTAT.

1 La elección se realiza como una base territorial apropiada para estudio de caso, en ningún momento debe entenderse una identificación entre GAL y Distrito Rural, no se agotan las posibilidades de contrastación para otras identidades territoriales administrativas o espacios delimitados por otras consideraciones funcionales $\mathrm{y} / \mathrm{o}$ políticas. 


\section{Metodología de Protosistemas Locales para la validación de los territorios de los Grupos de Acción Local, como demarcación geográfica apropiada para el distrito rural}

En Castilla-La Mancha se hallan $31 \mathrm{GAL}$, distribuidos en las cinco provincias, tal y como puede apreciarse en el Mapa 1 y el Cuadro 2.

$$
\text { MAPA } 1
$$

\section{GAL de Castilla-La Mancha}

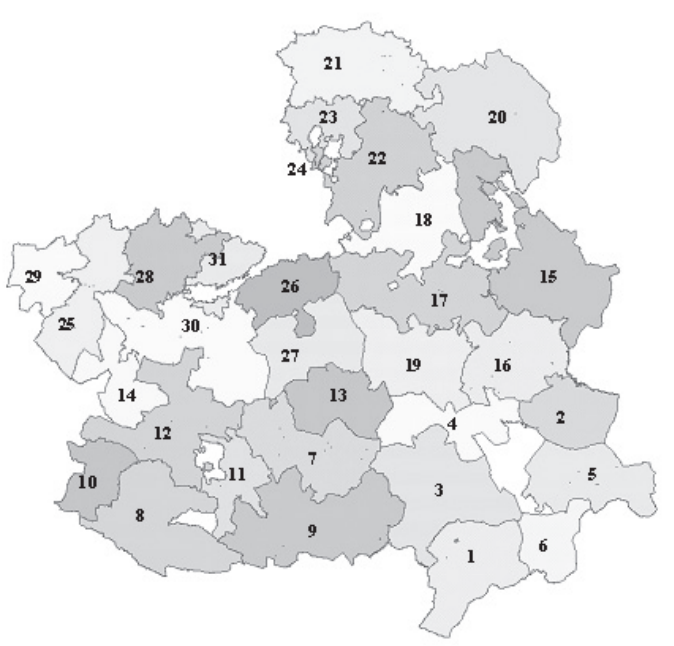

CUADRO 2

GAL de Castilla-La Mancha

\begin{tabular}{|c|c|c|c|c|}
\hline Albacete & Ciudad Real & Cuenca & Guadalajara & Toledo \\
\hline Sierra Segura (1) & $\begin{array}{l}\text { Alto Guadiana - } \\
\text { Mancha (7) }\end{array}$ & Prodese (15) & Molina de Aragon (20) & Ipeta (25) \\
\hline Manchuela (2) & Alcudia (8) & Adiman (16) & Adel (21) & Quijote (26) \\
\hline Sacam (3) & Tierras de Libertad (9) & Adesiman (17) & Fadeta (22) & Dulcinea (27) \\
\hline Mancha Jucar - Centro (4) & Montesur (10) & $\begin{array}{l}\text { Alcarria } \\
\text { Conquense (18) }\end{array}$ & Adac (23) & Castillos Medio Tajo (28) \\
\hline Monte Ibérico (5) & Calatrava (11) & Zancara (19) & Corredor de Henares (24) & Adecor (29) \\
\hline \multirow[t]{3}{*}{ Hellin (6) } & Montes Norte (12) & & & Montes de Toledo (30) \\
\hline & Promancha (13) & & & $\begin{array}{l}\text { La Sagra y Corredor } \\
\text { de Toledo (31) }\end{array}$ \\
\hline & Cabañeros (14) & & & \\
\hline
\end{tabular}

Fuente: Elaboración propia. 
Estos GAL derivan de la aplicación de la Iniciativa Comunitaria LEADER y del P.O. PRODER en sus distintas fases y, en la actualidad, de la aplicación del Eje 4 del FEADER. Fueron acordados en el Plan de Zonificación aprobado en la Cortes de Castilla-La Mancha en el año 2000 y es una clasificación que, como se demostrará con la metodología ISTAT, nos aproxima al nuevo concepto de desarrollo territorial; el Distrito Rural, que concentra a municipios con ciertas similitudes geográficas, sociales, históricas, económicas, culturales, que aglutinan puestos de trabajo y que ejercen una fuerza de atracción.

Aunque en el epígrafe anterior se presentó una primera aproximación para constatar el predominio de movimientos rural-rural en una dinámica centro-periferia, aquí se estudia desde la perspectiva de las tasas de variación. En concreto, comparando la dispersión de las tasas de variación de la población entre 1981 y 2009 de cada municipio con su población en 2009 y analizando la posición de la cabecera comarcal con el resto. Así, el planteamiento del Centro- Periferia Intrarural se verificaría cuando se cumpliesen alguna de las siguientes hipótesis:

H1: Centro recibe población y la periferia la pierde.

H2: Centro recibe más población que la periferia.

H3: Centro pierde menos población que la periferia.

H4: Centro recibe población y del resto, unos reciben (menos que el centro) $y$ otros pierden.

De los 31 GAL tan sólo Montesur (Ciudad Real) no cumplía las hipótesis, el resto sí verificó alguna de ellas: 13 GAL la Hipótesis 1(42\%), 9 GAL la Hipótesis 2 (29\%), 6 GAL la Hipótesis $3(19 \%)$ y 2 GAL la Hipótesis 4 (10\%), como se resumimos en el Cuadro 3. 


\section{CUADRO 3}

Hipótesis Centro (cabecera)-Periferia (resto municipios) Intrarural. 1981-2009

\begin{tabular}{|c|c|c|c|c|c|c|}
\hline Prov. & GAL & H1 & H2 & H3 & H4 & Ninguna \\
\hline $\mathrm{AB}$ & Sierra Segura & $\mathrm{X}$ & & & & \\
\hline $\mathrm{AB}$ & Manchuela & $\mathrm{X}$ & & & & \\
\hline $\mathrm{AB}$ & Sacam & & & $\mathrm{X}$ & & \\
\hline $\mathrm{AB}$ & Mancha Jucar - Centro & $\mathrm{X}$ & & & & \\
\hline $\mathrm{AB}$ & Monte Iberico & $\mathrm{X}$ & & & & \\
\hline $\mathrm{AB}$ & Hellin & $\mathrm{X}$ & & & & \\
\hline $\mathrm{CR}$ & Alto Guadiana - Mancha & & & & $\mathrm{X}$ & \\
\hline CR & Alcudia & & & $\mathrm{X}$ & & \\
\hline $\mathrm{CR}$ & Tierras De Libertad & $\mathrm{X}$ & & & & \\
\hline $\mathrm{CR}$ & Montesur & & & & & $\mathrm{X}$ \\
\hline CR & Calatrava & & & & $\mathrm{X}$ & \\
\hline $\mathrm{CR}$ & Montes Norte & $\mathrm{X}$ & & & & \\
\hline CR & Promancha & & $\mathrm{X}$ & & & \\
\hline CR & Cabañeros & & & $\mathrm{X}$ & & \\
\hline $\mathrm{CU}$ & Prodese & & & $\mathrm{X}$ & & \\
\hline $\mathrm{CU}$ & Adiman & $\mathrm{X}$ & & & & \\
\hline $\mathrm{CU}$ & Adesiman & $\mathrm{X}$ & & & & \\
\hline $\mathrm{CU}$ & Alcarria Conquense & & & $\mathrm{X}$ & & \\
\hline $\mathrm{CU}$ & Zancara & $\mathrm{X}$ & & & & \\
\hline GU & Molina De Aragon & & & $\mathrm{X}$ & & \\
\hline GU & Adel & $\mathrm{X}$ & & & & \\
\hline GU & Fadeta & & $\mathrm{X}$ & & & \\
\hline GU & Adac & & $\mathrm{X}$ & & & \\
\hline GU & Corredor De Henares & & $\mathrm{X}$ & & & \\
\hline TO & Ipeta & $\mathrm{X}$ & & & & \\
\hline TO & Quijote & & $\mathrm{X}$ & & & \\
\hline TO & Dulcinea & & $\mathrm{X}$ & & & \\
\hline TO & Castillos Medio Tajo & & $\mathrm{X}$ & & & \\
\hline TO & Adecor & $\mathrm{X}$ & & & & \\
\hline TO & Montes De Toledo & & $\mathrm{X}$ & & & \\
\hline TO & La Sagra Y Corredor De Toledo & & $\mathrm{X}$ & & & \\
\hline
\end{tabular}

Notas: Las casillas señaladas con una X son las que sí se cumple la hipótesis de centro-periferia. Fuente: Elaboración propia. 
En conclusión, este análisis confirma que la dinámica poblacional en el medio rural de Castilla-La Mancha crea nuevas concentraciones geográficas de población, cambiando el paisaje rural de los pueblos de la región y transformando la distribución demográfica en detrimento del medio rural más tradicional.

Pero esta tendencia, no sólo crea concentraciones geográficas de población, sino también de ocupados como se evidencia empíricamente, con diferentes métodos; entre ellos los indicadores econométricos de aglomeración local y global de Di Giacinto y Pagnini (2011). En este artículo se adaptará la metodología definida en 1996 por el Instituto Nacional de Estadística Italiano (ISTAT), basada en los algoritmos de los Protosistemas Locales (PSL). Con ellos se identificarán los municipios que concentran puestos de trabajo y que ejercen una fuerza de atracción. El algoritmo PSL para un municipio A, matemáticamente, se define como:

$$
P S L_{A}=\min \left[\frac{M C(A)}{0,75}, 1\right] * \min \left[\frac{W(A)}{1.000}, 1\right]
$$

Si $P S L_{A}>0.75$, A se considera un municipios concentrador de trabajadores activos. Si $P S L_{A} \leq 0.75$, A se considera un municipios no concentrador de trabajadores y son unificados en torno a municipios concentradores de trabajadores.

En la Eq. [2], A es el municipio en examen (probable concentrador), B son los municipios no $\mathrm{A}, W(A)$ son los ocupados que trabajan en $\mathrm{A}$ y residen en $\mathrm{A}$ o en $\mathrm{B}$, $R W(A)$ son los ocupados que residen y trabajan en $\mathrm{A}, R(A)$ son los ocupados que residen en A y trabajan en A o en $\mathrm{B}, \mathrm{y} M C(A)$ es un indicador de autocontención:

$$
M C(A)=\min \left[\frac{R W(A)}{W(A)}, \frac{R W(A)}{R(A)}\right]
$$

Para el calibrado del algoritmo se utilizan datos de movilidad laboral residenciatrabajo procedentes del Censo de Población de 2001 (INE), utilizando la información sobre población ocupada, población residente y desplazamientos por motivos laborales a poblaciones vecinas desde el lugar de residencia. Los resultados del algoritmo aplicado los resumimos en Cuadro 4. 


\section{CUADRO 4}

\section{Municipios atractores de población. Algoritmo de PSL}

\begin{tabular}{|c|c|c|c|}
\hline Prov. & GAL & Municipios que concentran trabajadores (PSL $>0,75$ ) & Coinciden cabecera GAL \\
\hline $\mathrm{AB}$ & Sierra Segura & Elche de la Sierra & Sí \\
\hline $\mathrm{AB}$ & Manchuela & Casas Ibáñez, Madrigueras, Villamalea & Sí \\
\hline $\mathrm{AB}$ & Sacam & Munera* & Sí \\
\hline $\mathrm{AB}$ & Mancha Jucar - Centro & Villarrobledo, La Roda, Tarazona & Sí \\
\hline $\mathrm{AB}$ & Monte Iberico & Almansa, Caudete, Montealegre, Chinchilla & Sí \\
\hline $\mathrm{AB}$ & Hellin & Hellín, Tobarra & Sí \\
\hline $\mathrm{CR}$ & Alto Guadiana - Mancha & Manzanares, Daimiel, La Solana & Sí \\
\hline $\mathrm{CR}$ & Alcudia & Almodóvar del Campo, Argamasilla de Calatrava & Sí \\
\hline $\mathrm{CR}$ & Tierras de Libertad & Valdepeñas, Villanueva de los Infantes & Sí \\
\hline $\mathrm{CR}$ & Montesur & Almadén & Sí \\
\hline $\mathrm{CR}$ & Calatrava & Miguelturra, Almagro, Bolaños & Sí \\
\hline $\mathrm{CR}$ & Montes Norte & Porzuna, Malagón, Piedrabuena & Sí \\
\hline $\mathrm{CR}$ & Promancha & $\begin{array}{l}\text { Tomelloso, Alcázar de San Juan, Campo de Criptana, } \\
\text { Socuéllamos, Herencia, Pedro Muñoz }\end{array}$ & Sí \\
\hline $\mathrm{CR}$ & Cabañeros & - & - \\
\hline $\mathrm{CU}$ & Prodese & - & - \\
\hline $\mathrm{CU}$ & Adiman & Quintanar del Rey, Motilla, Iniesta & Sí \\
\hline $\mathrm{CU}$ & Adesiman & Tarancón & Sí \\
\hline $\mathrm{CU}$ & Alcarria Conquense & Huete * & Sí \\
\hline $\mathrm{CU}$ & Zancara & Mota del Cuervo, San Clemente, Las Pedroñeras & Sí \\
\hline GU & Molina de Aragon & Molina de Aragón & Sí \\
\hline GU & Adel & Sigüenza & Sí \\
\hline GU & Fadeta & Brihuega* & Sí \\
\hline GU & Adac & - & - \\
\hline GU & Corredor de Henares & Azuqueca de Henares* & Sí \\
\hline TO & Ipeta & Talavera de la Reina, Cebolla & Sí \\
\hline TO & Quijote & Ocaña, Santa Cruz & Sí \\
\hline TO & Dulcinea & Quintanar de la Orden, Madridejos, Villacañas & Sí \\
\hline TO & Castillos Medio Tajo & Torrijos, Fuensalida, Puebla de Montalbán & Sí \\
\hline TO & Adecor & Calera y Cozas* & Sí \\
\hline TO & Montes de Toledo & Sonseca, Mora & Sí \\
\hline TO & La Sagra y Corredor de Toledo & Illescas, Seseña* & Sí \\
\hline
\end{tabular}

Notas: $(*)$ Municipios cabeceras de GAL con mayor PDL pero que no alcanzan el 0,75.

Fuente: Elaboración propia.

De la aplicación de la metodología ISTAT se deduce que: justamente, los municipios concentradores de trabajadores $(P S L>0,75)$ coinciden con las cabeceras de los GAL, que además son los municipios que más población concentran en el periodo 1981-2009 y se confirma el término Distrito Rural. En conclusión, con este método se diferencian los municipios que ejercen como ejes de sistemas regionales de tra- 
bajo de aquellos que lo hacen de periferias de dichos sistemas. Esta organización territorial obedece a la vinculación casi natural de municipios vecinos que, por sus vínculos, desarrollan flujos poblacionales, económicos y laborales y que, a su vez, sirven de base para adelantar programas mancomunados de inversión pública, mantenimiento ambiental, desarrollo vial, etc. Se asiste entonces al surgimiento de municipios que, por diferentes factores, se erigen como centros generadores de dinámicas productivas con un alcance mayor y que involucran municipalidades vecinas.

La verificación del surgimiento de estos Distritos Rurales retoma la figura de las tres fuerzas centrípetas Marshallianas, que son aplicables al caso en discusión de los fenómenos observados en el medio rural. Todos estos factores se refuerzan por la tendencia hacia la terciarización del aparato productivo acaecido en el medio rural, como expresa García Merino (2004): "En la actualidad la sociedad y la organización del espacio responden al mundo urbano, donde lo rural sólo es una forma de hábitat. Así, en los pueblos los servicios serán las actividades que ocupen a la mayor parte de la gente". A esto hay que añadir el avance de las comunicaciones, de las infraestructuras físicas y de los avances en la construcción de capital social, que van a ser la base para entender los movimientos de población en el medio rural, tal y como demostraremos en el epígrafe siguiente con los modelos hedónicos poblacionales.

\section{Razones de la dinámica de la población en los Distritos Rurales: un modelo hedónico}

Entre las razones de este punto de inflexión en la dinámica poblacional, Frey (1987) apunta la periodicidad, la reestructuración regional y descentralización. Esta última es la que ha tenido más acogida. Desde esta perspectiva el nuevo espacio productivo, determina las preferencias de localización residencial de trabajadores y consumidores. Otros investigadores señalan las desigualdades entre áreas geográficas como la principal causa, pero impulsada por los criterios individuales de los agentes. Las personas se trasladan hacia lugares donde esperan mejorar sus condiciones de vida, se responde así a los principios de racionalidad maximizadora: se busca maximizar beneficios reales, descontados los costos asociados al traslado. Por otro lado, Pérez $(1966,1971)$ ya hablaba del contraste entre mundos y situaciones; los menores ingresos de la actividad agrícola frente a los ingresos de las clases urbanas, las dificultades de acceso al consumo por la penuria en que se encontraban las redes de distribución del medio rural, el menor acceso a los servicios de información, sanitarios, de ocio y, principalmente, de enseñanza constituyen los elementos perceptibles de una situación dispar.

En resumen, el éxodo rural ha estado ligado a la actividad económica, pero esta visión no logra explicar todos los desplazamientos, ya lo señalaba Pérez (1966, 1971). Por ello, con el propósito de interpretar y describir los motivos de los flujos migratorios entre municipios, se plantean modelos que, agrupando los municipios de Castilla-La Mancha en el mismo esquema de los GAL, estudian el comportamiento de su población. En este sentido, son diversos los planteamientos teóricos estudiados: 
desde los modelos de desequilibrio, que parten de la hipótesis de que los movimientos migratorios responden, básicamente, a factores de naturaleza económica, hasta los modelos de equilibrio, desarrollados a partir de 1970, que consideraban las diferencias de tipo ambiental, comodidades o servicios locales y los factores compensadores respecto de las variables económicas.

En este artículo aplicamos la Teoría de los Modelos Hedónicos de Rosen como novedad metodológica al ser la más acorde a nuestra hipótesis de partida: la fuerza atractora de la población son los atributos locales, no las variables económicas. El resto de los modelos priorizan las diferencias económicas: los flujos migratorios se ven afectados, entre otras variables de naturaleza económica, por el crecimiento económico, el comportamiento de los salarios y el empleo.

La metodología inicial de Rosen se fundamenta en la relación existente entre el precio de mercado de un bien y las características que lo definen, de manera que es posible deducir el valor monetario de las mismas a partir de las diferencias observadas en el precio de mercado de los bienes que las incorporan. Comparando, los territorios son similares a cualquier bien al que se le aplique esta teoría; son heterogéneos y divisibles, se caracterizan por una serie de atributos individuales, cada uno de los cuales contribuiría a concentrar la población. El objetivo final es descubrir los atributos de un territorio que explican su población y asignar a cada característica la disposición marginal de una persona a residir en él.

Así se especificarán modelos que demuestren la adecuación de la hipótesis previa planteada, en el sentido que los aspectos económicos no son la razón principal de los desplazamientos de la población (que procede, básicamente, de la misma periferia comarcal), sino otros factores de índole social, como la oferta de servicios públicos (educación, sanidad, ocio, tecnologías de la información y comunicación) cuya trascendencia es admitida y que, como señala García-Arias (2004), son "el corpus doctrinal de la Economía". En nuestra investigación actúan de fuerzas centrípetas para la conformación de distritos rurales, en los que la terciarización en servicios productivos (de mercado) se produce a posteriori, generando la otra fuerza centrípeta de un Distrito Rural: las oportunidades de empleo, de inversión y el avance en los flujos de información y el proceso de modernización económica. Ambas fuerzas centrípetas se superponen a las centrifugas que puedan existir, conformando una concentración de población y tejido económico en lo que puede ser un centro rural a costa de la periferia de los núcleos próximos más pequeños y desfavorecidos. De modo intuitivo, Juste y Fernández (2005) ya afirman que "en general, los mayores niveles de diversificación productiva e industrial corresponden a los municipios, cabeceras de comarca de áreas rurales, con un nivel de población medio-bajo, habiendo mantenido o aumentado ligeramente su población en los últimos años". Esta percepción teórica se demostrará, cuantitativamente, estableciendo relaciones de causalidad, lo que permitirá avanzar con nuestra investigación.

La idea subyacente para aplicar la Teoría de los Modelos Hedónicos es, que si un municipio está constituido por un conjunto de servicios, su atractivo para la población será un agregado del valor individual de cada uno de ellos. Por tanto, con el objetivo de explicar la heterogeneidad inherente a un municipio, (servicios, actividad 
económica, localización), se ha considerado a éste en términos hedónicos. De tal manera, que la unidad (municipio) es conceptualizada no como bien homogéneo e indivisible, sino como una cesta de atributos individuales, cada uno de los cuales contribuye a concentrar la población. Este planteamiento responde a la teoría de los modelos hedónicos.

Diferentes estudios señalan a Court (1939) como el primer investigador en acuñar el término modelo hedónico, aunque el origen de sus aplicaciones resulta más controvertido; unos señalan a Haas (1922) y otros al propio Court (1939). Lo que no hay duda, es que la teoría de los modelos hedónicos se ha perfilado en el tiempo; así Lancaster (1966) desarrolló los fundamentos para estimar el valor de utilidad generado por las características de un bien y Rosen (1974) estableció el soporte teórico del modelo de precios hedónicos.

Simplificadamente, los modelos hedónicos establecen que los bienes están constituidos por un cierto número de atributos y que la decisión de un consumidor, en el proceso de maximización de su utilidad, al comprar un producto, viene afectada por ellos. Si extrapolamos esta idea al contexto poblacional, diremos que un modelo hedónico de población se referirá a la satisfacción que el individuo obtiene viviendo en un municipio según las características del mismo.

Dicho de otra manera, si se define $P_{i}$ como la población de un municipio $i$, ésta se puede describir por el vector $Q_{j, i}$ de los $k$ atributos de ese municipio $i$ :

$$
Q_{j, i}=\left(Q_{1 i}, Q_{2 i}, \ldots, Q_{k}\right)
$$

A partir de aquí se construye un modelo hedónico que explicite la relación funcional entre $P_{i}$ y sus respectivas características, $Q_{j, i}$, siendo su expresión:

$$
P_{i}=f\left(Q_{j}, \varepsilon_{i}\right)
$$

donde f es la relación funcional entre $P_{i}$ y $Q_{j, i}$, y $\varepsilon_{i}$ es la perturbación aleatoria.

Por otro lado, la función de utilidad de residir en un municipio $i, U_{i}$, dependerá tanto de $Q_{j, i}$ como de $P_{i}$. Analíticamente, su expresión es:

$$
U_{i}=U\left(P_{i}, Q_{j, i}\right)
$$

Por tanto, un individuo obtiene su satisfacción no directamente del municipio sino de los atributos de éste. Además, decidirá habitar en un municipio A frente a otro municipio B, si la utilidad que le proporcionen las características del primero (A) son mayores que las del segundo (B). En términos matemáticos sería:

$$
\frac{\partial U / \partial q_{j, A}}{\partial U / \partial A}>\frac{\partial U / \partial q_{j, B}}{\partial U / \partial B}
$$

En definitiva, los atributos $Q_{j, i}$ acercan a la población a un municipio y determinan la utilidad de vivir en él. Son fuerzas de atracción que cubren necesidades de interés comunitario, ofreciendo una mejor calidad de vida. En el contexto de Castilla- 
La Mancha, la provisión de luz, agua o teléfono son servicios más básicos y más universales, por lo que serían fuerzas de atracción: los servicios mínimos de sanidad ( $\left.Q_{\text {Sanidad }, i}\right)$, educación $\left(Q_{\text {Educación,i }}\right)$, tecnologías de la información y comunicación ( $\left.Q_{T I C, i}\right)$ y ocio $\left(Q_{O c i o, i}\right)$, dado que el resto estarían, en general, cubiertos. Quedaría por contrastar la influencia de cierto nivel de actividad económica $\left(Q_{\text {Economia }, i}\right)$.

Teniendo en cuenta cada $Q_{j, i}$ y sustituyéndolos en la ecuación (2), $P_{i}=f\left(Q_{j}, \varepsilon_{i}\right)$ se obtiene:

$$
P_{i}=f\left(Q_{\text {Sanidad }}, Q_{\text {Educación },} Q_{T I C}, Q_{\text {Ocio, }}, Q_{\text {Economia }}, \varepsilon_{i}\right)
$$

En donde, se establece como condición, que a medida que aumenten los servicios de un municipio, $Q_{j, i}$, su población, $P_{i}$, debiera aumentar. Matemáticamente, es la derivada parcial de la función $P_{i}=f\left(Q_{j}, \varepsilon_{i}\right)$, con respecto a cada uno de sus atributos:

$$
\frac{\partial P_{i}}{\partial Q_{j, i}}>0
$$

O lo que es lo mismo, si consideramos la ecuación [6], las derivadas parciales para cada uno de los atributos sería:

$$
\frac{\partial P_{i}}{\partial Q_{\text {Sanidad }}}>0, \frac{\partial P_{i}}{\partial Q_{\text {Educación }}}>0, \frac{\partial P_{i}}{\partial Q_{T I C}}>0, \frac{\partial P_{i}}{\partial Q_{\text {Ocio }}}>0, \frac{\partial P_{i}}{\partial Q_{\text {Economia }}}>0
$$

Por otro lado, la ecuación $P_{i}=f\left(Q_{j}, \varepsilon_{i}\right)$ presenta aspectos no completamente resueltos, como su forma funcional $f$. La forma funcional $f$ no necesariamente corresponde a una expresión lineal, debido a que la relación entre la población y las variables explicativas suele no serlo. Es decir, a medida que aumenta la cantidad de un atributo, la magnitud del impacto sobre la población no se calcula como una razón constante. Verdaderamente, la experiencia empírica demuestra que la relación entre la variable endógena $\left(P_{i}\right)$ y las variables exógenas $\left(Q_{j, i}\right)$ tiende a adoptar formas funcionales logarítmicas. De hecho, los primeros estudiosos ensayaron con funciones no lineales (logarítmica, semilogarítmica), seleccionándolas frente a las lineales. Siguiendo esta idea, se probará con cuatro funciones: Lineal, Logarítmica, Raíz cuadrada y Recíproca, que afectará sólo a la endógena $\left(P_{i}^{*}\right)$, ya que las variables exógenas son binarias.

Elegida la forma funcional del modelo, estimaremos por el Método de los Mínimos Cuadrados Ordinarios, procedimiento conocido que plantea utilizar, como estimación de los parámetros, aquella combinación de $(\beta i)$ que minimice la suma de los errores al cuadrado, $\sum_{i=1}^{n}\left(e_{i}\right)^{2}$, es decir, la suma de las diferencias al cuadrado entre el valor real y estimado de la endógena, que el modelo cometerá para las n observaciones disponibles:

$$
\min \sum_{i=1}^{n}\left(e_{i}\right)^{2}=\min \sum_{i=1}^{n}\left(P_{i}^{*}-\hat{P}_{i}^{*}\right)^{2}
$$


Así, aplicando Mínimos Cuadrados Ordinarios a la ecuación [4] resultará la siguiente ecuación estimada:

$$
P_{i}^{*}=\beta_{0}+\beta_{1} Q_{\text {Sanidad }, i}+\beta_{2} Q_{\text {Educación }}+\beta_{3} Q_{T I C}+\beta_{4} Q_{\text {Ocio }}+\beta_{5} Q_{\text {Economia }}
$$

que someteremos a evaluación, teniendo en cuenta los diferentes contrastes sobre estructura y perturbación aleatoria, hasta que la validemos estadística y econométricamente y podamos hacer interpretaciones económicas.

En definitiva, la metodología planteada consiste en construir un modelo econométrico que explicite la relación funcional entre la población de un municipio $P_{i}$ y sus respectivas características $Q_{j, i}$, dotarlo de información estadística, estimarlo y validarlo, procesando luego los resultados para valorar cada atributo.

\section{Un modelo hedónico poblacional para Castilla-La Mancha}

Se pretende determinar de entre los factores ya presentados sanidad ( $\left.Q_{\text {Sanidad }, i}\right)$, educación ( $\left.Q_{E \text { ducación, } i}\right)$, ocio $\left(Q_{O c i o, i}\right)$, tecnologías de la información y comunicación $\left(Q_{T I C, i}\right)$ y nivel de actividad económica $\left(Q_{E c o n o m i a, i}\right)$, los más influyentes en los movimientos poblacionales $\left(P_{i}\right)$ entre los municipios de un mismo GAL. De esta manera se plantean 31 modelos hedónicos poblacionales, uno por cada GAL, estudiando en cada uno de ellos como el siguiente:

$$
P_{i}^{*}=\beta_{0}+\beta_{1} Q_{\text {Sanidad }, i}+\beta_{2} Q_{\text {Educación }}+\beta_{3} Q_{T I C}+\beta_{4} Q_{\text {Ocio }}+\beta_{5} Q_{\text {Economia }}+\varepsilon_{i}[13]
$$

donde $\varepsilon_{i} \approx N\left(0, \sigma_{\varepsilon}^{2} I_{n}\right)$ es la perturbación aleatoria que sigue una distribución normal.

La ecuación [13] requiere de información estadística para ser estimada, la cual se ha conseguido de diversas fuentes y nos ha permitido conformar una base de datos de corte transversal con los 919 municipios de la región para $2008^{2}$. Las variables que se utilizan, su significado y su origen, las mostramos a continuación.

La variable $\left(P_{i}\right)$ representa la población de un municipio $i$. La fuente estadística es el Instituto de Estadística de Castilla-La Mancha. La variable es cuantitativa, pero a partir de ahora las variables que presentamos son cualitativas, en concreto, proxies que nos aproximan a las variables, objeto de análisis. Toman el valor " 1 " cuando se produce la característica mencionada y " 0 " si no se produce.

La primera variable cualitativa que presentamos es una proxy a un índice económico oportunidades de empleo en el medio rural $\left(Q_{\text {Economia }, i}\right)$ para medir la actividad económica y laboral de un municipio. Se plantea como el cociente entre los contratos indefinidos (CI) y la población potencialmente activa (PPA) en el municipio $i$. Analíticamente, se formula como:

$$
Q_{\text {Economia }, i}=\frac{C I_{i}}{P P A_{i}}=\left\{\begin{array}{l}
1 \\
0
\end{array}\right\}
$$

\footnotetext{
Se ha seleccionado este año, porque es el más reciente para el que hemos conseguido una base de datos más completa.
} 
que toma valor 1 cuando los contratos indefinidos per cápita activa de $i$ superen la media de contratos indefinidos per cápita activa del GAL. Analíticamente, su expresión es:

$$
Q_{\text {Economia } i}= \begin{cases}\text { si } \frac{C I_{i}}{P P A_{i}} \geq & \frac{\sum_{i=1}^{n}\left(\frac{C I_{i}}{P P A_{i}}\right)}{n} \\ 0 \text { si } & \frac{C I_{i}}{P P A_{i}}<\frac{\sum_{i=1}^{n}\left(\frac{C I_{i}}{P P A_{i}}\right)}{n}\end{cases}
$$

La fuente estadística de los CI es el Servicio Público de Empleo Estatal y de la PPA el Instituto de Estadística de Castilla-La Mancha.

Otra variable cualitativa es la que intenta aproximarnos a la calidad sanitaria de un municipio ( $\left.Q_{\text {Sanidad }, i}\right)$, medida por la existencia de centros de atención sanitaria. La clasificación que hace el Servicio de Salud de Castilla-La Mancha de centros de salud son: consultorios, centros de atención primaria y hospitales. En general, un individuo se siente más tranquilo, si en el lugar donde reside existe al menos un centro sanitario activo las 24 horas, es por ello, que hemos seleccionado, entre todos los centros de salud, los de atención primaria. Esta variable toma valores 1 y 0 , $Q_{\text {Educación, }}=\left\{\begin{array}{l}1 \\ 0\end{array}\right\}, 1$ sí el municipio dispone de ese tipo de centros y 0 en caso contrario.

La fuente estadística ha sido el Servicio de Salud de Castilla-La Mancha.

Los centros educativos son otra fuerza atractora de la población, porque es en ellos donde los niños/jóvenes complementan sus conocimientos y su base moral y social. Una clasificación de centros educativos es: colegios, institutos y universidad. Pero en este estudio ni los colegios ni la universidad son determinantes, los primeros porque se hallan en todos los municipios y los segundos porque sólo se encuentran en capitales de provincia, que no son municipios a incluirse en los GAL. Por lo tanto, son los institutos los únicos que nos sirven de indicadores. De este modo, la variable $Q_{\text {Educación, } i}$ toma valor 1 si el municipio dispone de al menos un instituto y valor 0 en caso de no hallarse ninguno, $Q_{\text {Educación, } i}=\left\{\begin{array}{l}1 \\ 0\end{array}\right\}$. La fuente estadística de donde se obtiene esta información es la Junta Comunidades de Castilla-La Mancha.

Por otro lado, en la actualidad son las Tecnologías de la Información y la Comunicación, y particularmente el acceso a Internet, condicionantes estructurales para la dinámica social y la económica. A partir de la base de datos de la Junta de Comunidades de Castilla-La Mancha obtenemos los habitantes con línea de internet ADSL $\left(P_{A D S L, i}\right)$, y a partir de aquí planteamos una proxy $\left(Q_{T I C, i}\right)$ como el cociente entre los habitantes con línea de internet ADSL en un municipio $\left(P_{A D S L, i}\right)$ y la población total de ese municipio $\left(P_{i}\right)$ : 


$$
Q_{T I C, i}=\frac{P_{A D S L, i}}{P_{i}}=\left\{\begin{array}{l}
1 \\
0
\end{array}\right\}
$$

La variable tomará el valor 1 cuando el cociente sea mayor o igual a 0,5 , es decir, cuando la mitad o más de la población de un municipio tenga acceso a internet y tomará el valor 0 cuando ni la mitad de la población tenga acceso a internet:

$$
Q_{T I C, i}=\left\{\begin{array}{lll}
1 & \text { si } & \frac{P_{A D S L, i}}{P_{i}} \geq 0,5 \\
0 & \text { si } \frac{P_{A D S L, i}}{P_{i}}<0,5
\end{array}\right.
$$

Por último, otra característica que hace atractivo a un municipio son los centros de ocio $\left(Q_{\text {Ocio }, i}\right)$. Hemos escogido como centros de ocio cines y teatros. La variable toma el valor 1 si el municipio dispone de un centro de ocio (teatro o cine) y 0 cuando no disponga de ellos. Los datos de los cines son del Ministerio de Cultura y los teatros de la Junta de Comunidades de Castilla-La Mancha.

\section{Resultados}

Como ya hemos señalado en el epígrafe anterior la primera tarea es averiguar la forma funcional de los modelos hedónicos. Se han considerado las siguientes formas funcionales:

\section{CUADRO 5}

\begin{tabular}{|c|c|}
\hline Función Funcional & Ecuación \\
\hline LINEAL (I) & $P_{i}=\beta_{0}+\beta_{1} Q_{\text {Sanidad }, i}+\beta_{2} Q_{\text {Educación }}+\beta_{3} Q_{T I C}+\beta_{4} Q_{\text {Ocio }}+\beta_{5} Q_{\text {Economia }}+\varepsilon_{i}$ \\
\hline \multicolumn{2}{|l|}{ NO LINEALES (2) } \\
\hline $\begin{array}{l}\text { Raiz cuadrada en endógena } \\
\text { (2a) }\end{array}$ & $\sqrt{P_{i}}=\beta_{0}+\beta_{1} Q_{\text {Sanidad }, i}+\beta_{2} Q_{E \text { ducación }}+\beta_{3} Q_{T I C}+\beta_{4} Q_{\text {Ocio }}+\beta_{5} Q_{E c o n o m i a}+\varepsilon_{i}$ \\
\hline Logaritmica-lineal (2b) & h $P_{i}=\beta_{0}+\beta_{1} Q_{\text {Sanidad }, i}+\beta_{2} Q_{E \text { ducación }}+\beta_{3} Q_{T I C}+\beta_{4} Q_{\text {Ocio }}+\beta_{5} Q_{E \text { conomia }}+\varepsilon_{i}$ \\
\hline $\begin{array}{l}\text { Reciproco raiz cuadrada en } \\
\text { endógena }(2 c)\end{array}$ & $1 / \sqrt{P_{i}}=\beta_{0}+\beta_{1} Q_{\text {Sanidad }, i}+\beta_{2} Q_{\text {Educación }}+\beta_{3} Q_{T I C}+\beta_{4} Q_{\text {Ocio }}+\beta_{5} Q_{E c o n o m i a}+\varepsilon_{i}$ \\
\hline Recíproco en endógena (2d) & $1 / P_{i}=\beta_{0}+\beta_{1} Q_{\text {Sanidad }, i}+\beta_{2} Q_{\text {Educación }}+\beta_{3} Q_{T I C}+\beta_{4} Q_{\text {Ocio }}+\beta_{5} Q_{\text {Economia }}+\varepsilon_{i}$ \\
\hline
\end{tabular}

\section{Forma Funcional}


Estimadas las cinco opciones de ecuaciones, por el método de Mínimos Cuadrados Ordinarios, para los 31 GAL, y comparando sus Sumas de Cuadrados Residuales, la mejor opción es la logarítmica, como ocurre en general en estudios empíricos de modelos hedónicos ${ }^{3}$. Después, para comprobar si el modelo cumple las Hipótesis Clásicas $^{4}$, analizamos la significación individual (t-Student), conjunta (F-Snedecor), hetorocedasticidad (test de White), autocorrelación (Breusch-Godfrey), bondad $\left(R^{2}\right)$; corregimos las contrariedades derivadas de la especificación y estimación y, de ser necesario, reestimamos los modelos, siendo los atributos que explicarían los movimientos de la población los expuestos en el Cuadro 6.

\section{CUADRO 6}

\section{Atributos significativos que influyen en la movilidad de la población*}

\begin{tabular}{|c|c|c|c|c|c|c|}
\hline Prov. & GAL & Economía & Sanidad & Educación & TIC & Ocio \\
\hline $\mathrm{AB}$ & Sierra Segura & & & $\mathrm{X}$ & & \\
\hline $\mathrm{AB}$ & Manchuela & $\mathrm{X}$ & $\mathrm{X}$ & & & $\mathrm{X}$ \\
\hline $\mathrm{AB}$ & Sacam & & $\mathrm{X}$ & $\mathrm{X}$ & & \\
\hline $\mathrm{AB}$ & Mancha Jucar - Centro & & $\mathrm{X}$ & $\mathrm{X}$ & & \\
\hline $\mathrm{AB}$ & Monte Iberico (Corr Almansa) & $\mathrm{X}$ & & $\mathrm{X}$ & & $\mathrm{X}$ \\
\hline $\mathrm{AB}$ & Hellin (Campos de) & & $\mathrm{X}$ & $\mathrm{X}$ & & \\
\hline $\mathrm{CR}$ & Alto Guadiana - Mancha & & $\mathrm{X}$ & $\mathrm{X}$ & & \\
\hline $\mathrm{CR}$ & Alcudia (Valle de) & & & $\mathrm{X}$ & & $\mathrm{X}$ \\
\hline CR & Tierras de Libertad & & $\mathrm{X}$ & $\mathrm{X}$ & & $\mathrm{X}$ \\
\hline $\mathrm{CR}$ & Montesur (Comarca Almadén) & & $\mathrm{X}$ & & & \\
\hline CR & Calatrava (Campo de) & & $\mathrm{X}$ & & & \\
\hline $\mathrm{CR}$ & Montes Norte & & & $\mathrm{X}$ & & \\
\hline $\mathrm{CR}$ & Promancha (Mancha Norte) & & $\mathrm{X}$ & & & \\
\hline $\mathrm{CR}$ & Cabañeros & $\mathrm{X}$ & & $\mathrm{X}$ & & \\
\hline $\mathrm{CU}$ & Prodese & & $\mathrm{X}$ & & $\mathrm{X}$ & \\
\hline $\mathrm{CU}$ & Adiman & & & $\mathrm{X}$ & $\mathrm{X}$ & \\
\hline $\mathrm{CU}$ & Adesiman & & $\mathrm{X}$ & $\mathrm{X}$ & $\mathrm{X}$ & $\mathrm{X}$ \\
\hline $\mathrm{CU}$ & Alcarria Conquense & & $\mathrm{X}$ & & $\mathrm{X}$ & $\mathrm{X}$ \\
\hline $\mathrm{CU}$ & Zancara & & $\mathrm{X}$ & & $\mathrm{X}$ & $\mathrm{X}$ \\
\hline GU & Molina de Aragon & & $\mathrm{X}$ & $\mathrm{X}$ & $\mathrm{X}$ & \\
\hline GU & Adel & & & $\mathrm{X}$ & $\mathrm{X}$ & $\mathrm{X}$ \\
\hline GU & Fadeta & & $\mathrm{X}$ & $\mathrm{X}$ & $\mathrm{X}$ & \\
\hline GU & Adac & & & $\mathrm{X}$ & $\mathrm{X}$ & \\
\hline
\end{tabular}

Ver Cuadro 7 del anexo.

Ver Cuadro 8 del anexo. 


\section{CUADRO 6 (CONT.)}

Atributos significativos que influyen en la movilidad de la población*

\begin{tabular}{|c|c|c|c|c|c|c|}
\hline Prov. & GAL & Economía & Sanidad & Educación & TIC & Ocio \\
\hline GU & Corredor de Henares & & $\mathrm{X}$ & & & \\
\hline TO & Ipeta & $\mathrm{X}$ & & $\mathrm{X}$ & & $\mathrm{X}$ \\
\hline TO & Quijote & & $\mathrm{X}$ & & & \\
\hline TO & Dulcinea & & & $\mathrm{X}$ & & \\
\hline TO & Castillos del Medio Tajo & & $\mathrm{X}$ & $\mathrm{X}$ & $\mathrm{X}$ & \\
\hline TO & Adecor & & & $\mathrm{X}$ & $\mathrm{X}$ & \\
\hline TO & Montes de Toledo & & & $\mathrm{X}$ & & $\mathrm{X}$ \\
\hline TO & La Sagra y Corredor de Toledo & $\mathrm{X}$ & & $\mathrm{X}$ & & \\
\hline
\end{tabular}

*Ver resultados numéricos Cuadro 9 del anexo.

Fuente: Elaboración propia.

Analizando los resultados del cuadro se deduce que, a nivel individual, el atributo de la educación, $Q_{E \text { ducación, } i}$, es el factor que más incide para explicar la dinámica de la población de Castilla-La Mancha con presencia en el 71\% de los GAL; le sigue el factor sanidad $Q_{\text {Sanidad,i }}$ con una ponderación del 58\%; el factor tecnologías de la información y comunicación, $Q_{T I C, i}$ de peso en el 35,5\% de los GAL, el atributo ocio $Q_{O \text { cio }, i}$ con un $32 \%$ y, en último lugar, la economía (oportunidades de empleo) $Q_{\text {Economia }, i}$ con tan solo un $16 \%$, como se visualiza en la Gráfico 2:

\section{GRÁFICO 2}

\section{Atributos significativos ponderados sobre la movilidad de la población}

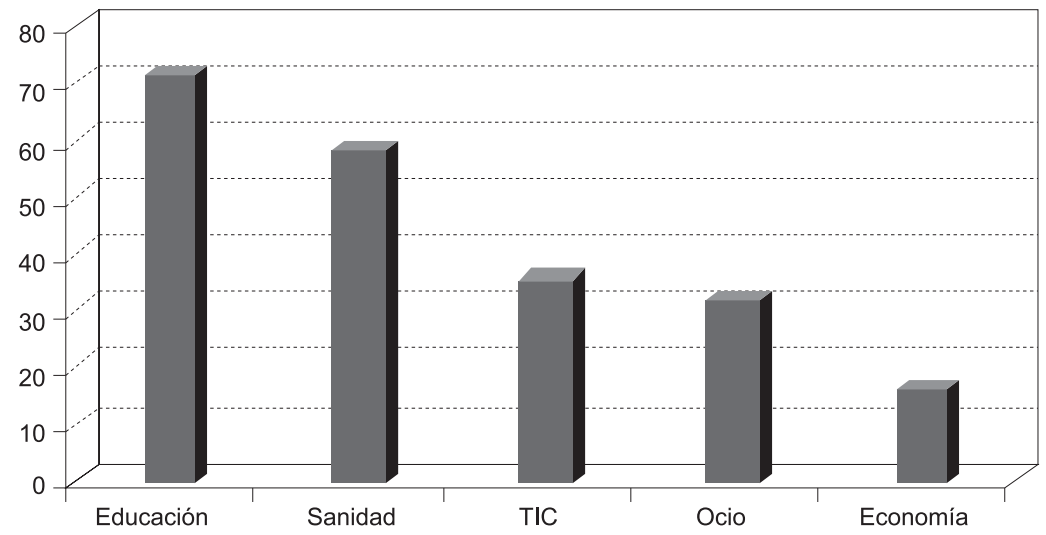

Fuente: Elaboración propia. 
Ahora bien, en el conjunto de los atributos $Q_{j, i}$, los resultados muestran que, en general en los GAL, ningún factor se presenta como único determinante en el arrastre de la población, sino la combinación de al menos dos factores, en los que el denominador común es sanidad y/o educación. Otro aspecto a destacar, es que en las provincias de Cuenca y Guadalajara es donde más se valora el acceso a las Tecnologías de la Información y Comunicación. Por último, señalar que el factor económico no es el detonante en los movimientos poblacionales entre municipios pertenecientes a un mismo GAL. Tan sólo en 4 de los 31 GAL resulta significativo el atributo, $Q_{\text {Economia }, i}$ : en La Manchuela (Albacete), Monte Ibérico (Albacete), Cabañeros (Ciudad Real), La Sagra (Toledo).

En los resultados establecidos queda confirmada la hipótesis de que el surgimiento de actividades terciarias públicas y de servicios básicos de no-mercado (educación, sanidad, nuevas tecnologías y centros de ocio) en el medio rural, sería la fuerza de atracción para la población, que a su vez llevaría, a posteriori, a un aumento de la actividad económica y reproduciría, en un nivel distintivo, fenómenos similares, pero de base productiva en lugar de social, que ya habían sido explicitados formalmente en los estudios sobre clúster y distritos industriales, conformándose así un nuevo concepto en el desarrollo rural: el Distrito Rural. Es estructura y ente que se presenta en las comarcas rurales y explica la dinámica de población y la base económica de los núcleos rurales en espacios homogéneos, decantando en una gama de sistemas de centros-periferia rurales y que condiciona la tradicional forma de entender las bases de la política de desarrollo territorial, en sus dimensiones rural y local.

\section{Conclusiones}

El cambio cualitativo de los movimientos poblacionales de Castilla-La Mancha se manifiesta a partir de 1980 en los núcleos rurales más grandes, donde se concentra la población absorbida de los núcleos periféricos, proceso que se ha acelerado en la década de los 2000. Se conforma así un "distrito social" que denomínanos Distrito Rural, por su proximidad conceptual a una redefinición del distrito industrial en las zonas rurales, y que se convierte en un nuevo concepto a tener en cuenta para unas nuevas bases del desarrollo territorial.

La demarcación territorial que se aproxima a la esencia de la definición del Distrito Rural, es el Grupo de Acción Local; ambos términos se identifican por la concentración de población, como evidencian las variaciones residenciales, y por la concentración de trabajadores, como se demuestra con la metodología Istat. Aglomeración que se da en una zona natural con características geográficas similares y semejanzas en sus modos de vida y culturales. Esta génesis de un sistema centro-periferia intrarural viene derivada, directamente, de la presencia de economías de escala sociales, presente en la dotación de infraestructuras básicas en estas poblaciones, que adquieren esas fuerzas centrípetas (en sentido de las aglomeraciones y la causalidad acumulativa de la geografía económica de Krugman). Desde este enfoque, los ámbitos rurales centrales se revalorizan y son sus ventajas residenciales y sociales los que 
motivan a los individuos a residir en unos frente a otros núcleos periféricos. A ello se añade que la terciarización de servicios de mercado rural es subsecuente a la dinámica demográfica, como se avanza con los Modelos Hedónicos Poblacionales que se han adaptado a estos efectos, como novedad metodológica para validar la hipótesis inicial del particular cambio demográfico rural.

Surge la duda de si las conclusiones serían ampliables a otras regiones de España o incluso al resto de la Unión Europea. Elegir Castilla-La Mancha como estudio de caso permite también inferir que la tipología y el hecho no es vinculable a una tipicidad regional y, por tanto, puede vehicularse a toda España, por lo que la investigación se está ampliando al caso de la totalidad del territorio de España, y los primeros resultados están confirmando la validez de las premisas y conclusiones alcanzadas para el caso manchego. En algunos estudios previos ya se ha señalado que el desarrollo de los mecanismos de servicios, información y de innovación tecnológica y, por ende, su impacto sobre el mercado de trabajo, han generado nuevas formas de poblamiento en las zonas rurales. Aun así, la falta de un desarrollo formal y los sesgos que presentan, los hacen incompletos y faltos de sistematización que es lo que se pretende subsanar con nuestra aportación.

Según los resultados que se desprenden de los modelos hedónicos poblacionales diseñados y planteados para el periodo de la última década, se concluye que la actividad económica ya no es la principal razón de los movimientos migratorios sino la disposición de servicios sociales básicos, sobre todo sanitarios y de educación, con incidencia de las NTIC y las ofertas culturales y de ocio. La presencia de rendimientos crecientes pasa del sector privado al público en forma de economías de escala sociales, siendo la función de producción territorial dominada por los factores de provisión de bienes públicos.

En efecto, cuando los efectos administrativos (ser cabecera comarcal) o de previa concentración de población están presentes, las fuerzas que se desencadenan generan una osmosis a la terciarización de sesgo privada, complementaria a la pública, que a su vez conforma nuevas oportunidades y ejerce de fuerza centrípeta. Valoremos que una importante ventaja de la especialización terciaria (sin paso previo por la fase de industrialización) es que proporciona diversas fuentes de empleo para la mayor parte de la población activa, e incluso en las zonas eminentemente rurales, la mayoría de los puestos de trabajo se crean en este sector que supone dos tercios del PIB de un territorio, en la actualidad. Por otro lado, las desigualdades geográficas, en peso del sector terciario de no mercado, generan desequilibrios espaciales en cuanto al nivel de vida (acceso a la educación y a la asistencia medico-sanitaria, posibilidades de acceder a los servicios recreativos y culturales) y configuran desequilibrios sistémicos que son restricciones a priori y a posteriori; por tanto son origen y mecanismos de causalidad acumulativa del proceso. Se genera un verdadero centro-periferia como enclave sistémico de procesos de aglomeración donde el centro es la cabecera comarcal, dotada de servicios públicos o bien el núcleo de mayor población, y la periferia son los núcleos de pequeña dimensión que no pueden acceder a la provisión de servicios públicos mínimos, generando el microproceso migratorio hacia el centro. 
Al margen de la constatación efectuada y del encuadre teórico del nuevo concepto de Distrito Rural, conviene tener presente las implicaciones para la política de desarrollo rural de nuestra investigación: valorar si la sedimentación de un sistema centro comarcal- periferia satelizado es un modelo que debe valorarse a aceptar o a combatir, dado que uno de los objetivos principales de la política de desarrollo rural es fijar y mantener la población en el medio rural. En el sistema centro-periferia rural, la población puede mantenerse en el medio rural, pero hay una restructuración y resituación espacial con un desequilibrio para las zonas rurales más periféricas y desfavorecidas. La dotación de infraestructuras se convierte en un eje distinto al papel, tradicionalmente asignado y el coste de oportunidad a asumir debe ser acometido con el correspondiente análisis coste-beneficio. Esta es una de las nuevas líneas de trabajo a desarrollar, pero lo que es indudable es que la política de desarrollo territorial, y dentro de ella la política de desarrollo rural, tiene un nuevo reto y una necesidad ineludible de redefinirse y reorientarse, pues la presencia de los distritos rurales es una realidad que debe configurar un eje central en la valoración de nuevos objetivos e instrumentos de esta política pública. También, puede valorarse que las coyunturas, en especial la actual crisis económica, pueden alterar las bases de las fuerzas que interactúan y que propician estos aglomerados rurales. Todo parece indicar que las movilidades demográficas vinculadas a la actividad económica pueden aumentar transitoriamente, pero no se configurarán como predominantes frente a las economías de escala sociales en los núcleos intermedios y grandes rurales y las zonas urbanas tampoco son referencia de potencialidad tractora como pueden haberlo sido en el pasado.

\section{Referencias}

Boix, V. y Galletto, R. (2005). Identificación de Sistemas Locales de Trabajo y Distritos Industriales en España. MITYC, Madrid.

Bielza de Ory, V. (2003). "La delimitación comarcal de Aragón”. En Infante, J. (Ed.): Las comarcas de Aragón: territorio y futuro. DGA-Presidencia y Relaciones Institucionales, Zaragoza: 115-136.

Court, A.T. (1939). The dynamics of automobile demand. General Motors, New York.

Di Giacinto, P. y Pagnini, M. (2011). "Local and global agglomeration patterns: Two econometrics-based indicators". Regional Science and Urban Economics, 41(3): 266-280.

Frey, W. (1987). "Migration and depopulation of the metrópolis: Regional restructuring or rural renaissance?" American Sociological Review, 52(2): 240-257.

García-Arias, J. (2004). "Un nuevo marco de análisis para los bienes públicos: La teoría de los bienes públicos globales". Revista Estudios de Economía Aplicada, 22(2): 187-212. 
García, L.V. (2004). "Tendencias en la evolución de los núcleos de población no urbanos hacia la mitad del s. XXI". En Nogués, S. (Ed.): El futuro de los espacios rurales. Universidad de Cantabria, Santander: 78-87.

Haas, G.C. (1922). "Sale Prices as a Basis for Farm Land Appraisal". Technical Bulletin, 9. Agricultural Experiment Station, University of Minnesota.

Juste, J.J. y Fernández. J.E. (2005). "Sistemas productivos locales en Castilla y León: Identificación y rasgos básicos". Revista Estudios Economía Aplicada, 23(2): 423-50.

Krugman, P. (1991). "Increasing returns and Economic Geography”. Journal of Political Economy, 99(3): 183-199.

Krugman, P. (1992). Geografía y Comercio. Antoni Bosch, Barcelona.

Lancaster, K.J. (1966). "A new approach to consumer theory". Journal of Political Economy, 74(2): 132-57.

Pérez, V. (1966). Estructura social del campo y del éxodo rural. Tecnos, Madrid.

Pérez, V. (1971). Emigración y cambio social: procesos migratorios y vida rural en Castilla. Ariel, Barcelona.

Rosen, S. (1974). "Hedonic prices and implicit markets: product differentiation in pure competition". Journal of Political Economy, 82(1): 34- 55. 


\section{ANEXO}

\section{CUADRO 7}

\section{Elección de función según la suma de cuadrados residual}

\begin{tabular}{|c|c|c|c|c|c|c|}
\hline Prov. & GAL & (1) & (2a) & (2b) & $(2 \mathrm{c})^{* *}$ & $(2 d)^{* *}$ \\
\hline $\mathrm{AB}$ & Sierra Segura & 528.378 & 627,8 & 1,5 & 0 & 0 \\
\hline $\mathrm{AB}$ & Manchuela & 6.879 .947 & 932,8 & 5,9 & 0 & 0 \\
\hline $\mathrm{AB}$ & Sacam & 4.954 .352 & 851,3 & 5,0 & 0 & 0 \\
\hline $\mathrm{AB}$ & Mancha Jucar - Centro & $1,41 \mathrm{E}+08$ & $2.388,1$ & 1,3 & 0 & 0 \\
\hline $\mathrm{AB}$ & Monte Iberico & 28.078 .335 & $1.131,4$ & 0,8 & 0 & 0 \\
\hline $\mathrm{AB}$ & Hellin* & $2,64 \mathrm{E}+08$ & $3.730,0$ & 0,9 & 0 & 0 \\
\hline $\mathrm{CR}$ & Alto Guadiana - Mancha & 78.680 .988 & $1.739,3$ & 1,3 & 0 & 0 \\
\hline CR & Alcudia & 5.334 .795 & 313,0 & 0,6 & 0 & 0 \\
\hline $\mathrm{CR}$ & Tierras de Libertad & 10.813 .115 & $1.382,0$ & 4,2 & 0 & 0 \\
\hline CR & Montesur & 2.005 .546 & 487,5 & 2,3 & 0 & 0 \\
\hline CR & Calatrava & 22.733 .364 & $2.091,0$ & 8,0 & 0 & 0 \\
\hline $\mathrm{CR}$ & Montes Norte & 8.270 .052 & $1.557,0$ & 6,8 & 0 & 0 \\
\hline $\mathrm{CR}$ & Promancha & $5,49 \mathrm{E}+08$ & $6.717,5$ & 1,4 & 0 & 0 \\
\hline $\mathrm{CR}$ & Cabañeros & 1.220 .378 & 199,0 & 0,5 & 0 & 0 \\
\hline $\mathrm{CU}$ & Prodese & 1.881 .666 & $1.394,4$ & 28,0 & 0 & 0 \\
\hline $\mathrm{CU}$ & Adiman & 28.218 .855 & $2.707,7$ & 14,0 & 0 & 0 \\
\hline $\mathrm{CU}$ & Adesiman & $1,17 \mathrm{E}+08$ & $5.752,0$ & 27,2 & 0 & 0 \\
\hline $\mathrm{CU}$ & Alcarria Conquense & 460.371 & 686,7 & 25,0 & 0 & 0 \\
\hline $\mathrm{CU}$ & Zancara & 10.010 .526 & $1.269,0$ & 9,3 & 0 & 0 \\
\hline GU & Molina de Aragon & $293.663,7$ & 649,3 & 35,0 & 0 & 0 \\
\hline GU & Adel & 14.640 .093 & $2.138,1$ & 35,2 & 0 & 0 \\
\hline GU & Fadeta & 9.045 .610 & $3.437,0$ & 44,6 & 0 & 0 \\
\hline GU & Adac & 27.147 .815 & $2.902,0$ & 25,0 & 0 & 0 \\
\hline GU & Corredor de Henares & $* * *$ & $* * *$ & $* * *$ & $* * *$ & $* * *$ \\
\hline TO & Ipeta & $4,10 \mathrm{E}+09$ & $35.164,9$ & 22,0 & 0 & 0 \\
\hline TO & Quijote & 20.844 .513 & $2.590,0$ & 7,4 & 0 & 0 \\
\hline TO & Dulcinea & 39.645 .646 & $1.940,0$ & 3,0 & 0 & 0 \\
\hline TO & Castillos Medio Tajo & 72.508 .081 & $6.549,0$ & 19,8 & 0 & 0 \\
\hline TO & Adecor & 11.435 .160 & $1.709,6$ & 5,8 & 0 & 0 \\
\hline TO & Montes de Toledo & 38.189 .899 & $3.332,0$ & 14,5 & 0 & 0 \\
\hline TO & La Sagra y Corredor de Toledo & $1,62 \mathrm{E}+08$ & $5.104,0$ & 4,2 & 0 & 0 \\
\hline
\end{tabular}

Notas: (*) Se escogen los modelos con menor Suma de Cuadrados Residual. (**) Las últimas dos columnas, aunque las sumas obtenidas son muy próximas a cero, no se tienen en cuenta porque, en general, los coeficientes estimados son negativos para atraer a la población. $(* * *)$ El Corredor de Henares no lo hemos podido estimar porque todos los municipios que lo integran disponen se de los servicios mínimos.

Fuente: Elaboración propia. 


\section{CUADRO 8}

\section{Factores $Q_{i, j}$ que influyen en la población. Probabilidad asociada a la t Student (se consideran significativos al $90 \%$ )}

\begin{tabular}{|c|c|c|c|c|c|c|}
\hline Prov. & GAL & ECONOMÍA & SANIDAD & EDUCACIÓN & TIC & OCIO \\
\hline $\mathrm{AB}$ & Sierra Segura & - & - & 0,014 & - & - \\
\hline $\mathrm{AB}$ & Manchuela & 0,03 & 0,06 & 0,14 & 0,11 & 0,01 \\
\hline $\mathrm{AB}$ & Sacam & 0,25 & 0,009 & 0,04 & & 0,35 \\
\hline $\mathrm{AB}$ & Mancha Jucar - Centro & 0,64 & 0,02 & 0,10 & _ & 0,45 \\
\hline $\mathrm{AB}$ & Monte Iberico & 0,028 & _- & 0,004 & _ & 0,03 \\
\hline $\mathrm{AB}$ & Hellin* & - & 0,16 & 0,14 & - & - \\
\hline $\mathrm{CR}$ & Alto Guadiana - Mancha & 0,94 & 0,010 & 0 & - & 0,79 \\
\hline $\mathrm{CR}$ & Alcudia & 0,31 & 0,33 & 0,008 & 0,15 & 0,02 \\
\hline $\mathrm{CR}$ & Tierras de Libertad & 0,41 & 0,08 & 0,003 & - & 0,005 \\
\hline $\mathrm{CR}$ & Montesur & - & 0,033 & - & - & - \\
\hline $\mathrm{CR}$ & Calatrava & 0,13 & 0,014 & - & - & 0,17 \\
\hline CR & Montes Norte & - & - & 0,04 & - & - \\
\hline $\mathrm{CR}$ & Promancha & - & 0,0055 & - & - & - \\
\hline $\mathrm{CR}$ & Cabañeros & 0,07 & - & 0,02 & - & - \\
\hline $\mathrm{CU}$ & Prodese & 0,53 & 0,0048 & 0,45 & 0,0004 & 0,37 \\
\hline $\mathrm{CU}$ & Adiman & 0,85 & - & 0,0005 & 0,0085 & 0,24 \\
\hline $\mathrm{CU}$ & Adesiman & 0,83 & 0,08 & 0,005 & 0,0032 & 0,04 \\
\hline $\mathrm{CU}$ & Alcarria Conquense & - & 0,016 & & 0,0006 & 0,02 \\
\hline $\mathrm{CU}$ & Zancara & - & 0,0095 & & 0,0032 & 0,04 \\
\hline GU & Molina de Aragon & 0,67 & 0,04 & 0,03 & 0,0002 & - \\
\hline GU & Adel & 0,41 & 0,25 & 0,0012 & 0,037 & 0,015 \\
\hline GU & Fadeta & - & 0,08 & 0 & 0,002 & \\
\hline GU & Adac & - & - & 0,02 & 0,07 & \\
\hline GU & Corredor de Henares & - & - & - & - & - \\
\hline TO & Ipeta & 0,13 & - & 0,02 & - & 0,0005 \\
\hline TO & Quijote & - & 0,0152 & - & - & - \\
\hline TO & Dulcinea & 0,78 & 0,50 & 0,0013 & - & 0,43 \\
\hline TO & Castillos Medio Tajo & - & 0,17 & 0,04 & 0,09 & - \\
\hline TO & Adecor & - & 0,15 & 0,0041 & 0,02 & - \\
\hline TO & Montes de Toledo & 0,43 & 0,20 & 0,0084 & - & 0,0003 \\
\hline TO & La Sagra y Corredor de Toledo & 0,0018 & - & 0,0003 & - & 0,19 \\
\hline
\end{tabular}

Notas: Las casillas sombreadas son las significativas.

(a) No todas las casillas aparecen con valor de probabilidad porque ha sido necesario rehacer el modelo, omitiendo variables que no resultaban significativas.

(b) El Corredor de Henares no lo hemos podido estimar porque todos los municipios que lo integran disponen de los servicios mínimos.

Fuente: Elaboración propia. 
CUADRO 9

Resumen de hipótesis clásicas

\begin{tabular}{|c|c|c|c|c|c|}
\hline Prov. & GAL & $\begin{array}{c}\text { Significatividad } \\
\text { Ccnjunta F Snedecor } \\
(\mathrm{p}<0,10)\end{array}$ & $\begin{array}{l}\text { Ausencia heterocedasticidad } \\
\text { contraste de White para sec- } \\
\text { ción cruzada }(p>0,10)\end{array}$ & $\begin{array}{l}\text { Ausencia Autocorrelacion } \\
\text { Breusch-Godfrey }(p>0,10)\end{array}$ & $\begin{array}{l}\text { R2 } \\
\%\end{array}$ \\
\hline $\mathrm{AB}$ & Sierra Segura & 0,0033 & 0,56 & 0,25 & 60 \\
\hline $\mathrm{AB}$ & Manchuela & 0 & 0,57 & 0,28 & 80 \\
\hline $\mathrm{AB}$ & Sacam & 0 & 0,45 & 0,72 & 73 \\
\hline $\mathrm{AB}$ & Mancha Jucar - Centro & 0,01 & - & 0,99 & 94 \\
\hline $\mathrm{AB}$ & Monte Iberico & 0 & 0,3 & 0,48 & 90 \\
\hline $\mathrm{AB}$ & Hellin & 0,13 & - & 0,08 & 86 \\
\hline CR & Alto Guadiana - Mancha & 0 & 0,08 & 0,08 & 94 \\
\hline CR & Alcudia & 0 & - & 0,84 & 94 \\
\hline $\mathrm{CR}$ & Tierras Libertad & 0 & 0,20 & 0,43 & 80 \\
\hline CR & Montesur & 0,033 & 0,90 & 0,97 & 55 \\
\hline CR & Calatrava & 0,006 & 0,23 & 0,49 & 73 \\
\hline $\mathrm{CR}$ & Montes Norte & 0,008 & 0,41 & 0,26 & 52 \\
\hline $\mathrm{CR}$ & Promancha & 0,0055 & 0,23 & 0,13 & 81 \\
\hline $\mathrm{CR}$ & Cabañeros & 0,01 & 0,18 & 0,21 & 82 \\
\hline $\mathrm{CU}$ & Prodese & 0 & 0,85 & 0,15 & 52 \\
\hline $\mathrm{CU}$ & Adiman & 0 & 0,75 & 0,77 & 81 \\
\hline $\mathrm{CU}$ & Adesiman & 0 & 0,94 & 0,41 & 62 \\
\hline $\mathrm{CU}$ & Alcarria Conquense & 0 & 0,47 & 0,22 & 56 \\
\hline $\mathrm{CU}$ & Zancara & 0 & 0,45 & 0,41 & 63 \\
\hline GU & Molina de Aragon & 0 & 0,91 & 0,55 & 50 \\
\hline GU & Adel & 0 & 0,95 & 0,89 & 58 \\
\hline GU & Fadeta & 0 & 0,56 & 0,77 & 60 \\
\hline GU & Adac & 0 & 0,36 & 0,93 & 75 \\
\hline GU & Corredor de Henares & & & & \\
\hline TO & Ipeta & 0 & 0,16 & 0,54 & 67 \\
\hline TO & Quijote & 0,015 & 0,23 & 0,20 & 35 \\
\hline TO & Dulcinea & 0,0014 & 0,55 & 0,16 & 78 \\
\hline TO & Castillos Medio Tajo & 0 & 0 & 0,34 & 65 \\
\hline TO & Adecor & 0 & 0,39 & 0,19 & 66 \\
\hline TO & Montes de Toledo & 0 & 0,91 & 0,02 & 71 \\
\hline TO & La Sagra y Corredor de Toledo & 0 & 0,07 & 0,21 & 59 \\
\hline
\end{tabular}

Fuente: Elaboración propia. 
